\title{
SMOOTHNESS OF THE SINGULAR LIMIT OF INERTIAL MANIFOLDS OF SINGULARLY PERTURBED EVOLUTION EQUATIONS
}

\author{
DANIEL ŠEVČOVIČ \\ Institute of Applied Mathematics, Comenius University, Mlynská dolina, 842 15, Bratislava, Slovak Republic
}

(Received 23 May 1994; received for publication 7 July 1995)

Key words and phrases: Inertial manifolds, smoothness of the singular limit, constitutive models of shearing motions.

\section{INTRODUCTION}

The aim of this paper is to investigate the singular limit behavior of incrtial manifolds of the following singularly perturbed system of evolution equations in Banach spaces

$$
\begin{aligned}
U_{t} & =G_{\varepsilon}(U, S) \\
\varepsilon S_{t}+A S & =F_{\varepsilon}(U, S),
\end{aligned}
$$

where $\varepsilon \geq 0$ is a small parameter, $X, Y$ are Banach spaces, $A$ is a sectorial operator in a Banach space $Y, Y^{\alpha}$ is the fractional power space and $F_{\varepsilon}: X \times Y^{\alpha} \rightarrow Y ; G_{\varepsilon}: X \times Y^{\alpha} \rightarrow X$; are smooth bounded functions, $\alpha \in[0,1), F_{\varepsilon} \rightarrow F_{0}, G_{\varepsilon} \rightarrow G_{0}$ as $\varepsilon \rightarrow 0^{+}$. It is well known that the above system of equations generates a $C^{1}$ semi-flow $S_{\varepsilon}$ in the phase-space $X \times Y^{\alpha}$ for any $\varepsilon>0$ (cf. Henry [1]). According to Marion [2] the semi-flow $S_{\varepsilon}$ possesses an invariant attracting manifold $\mathfrak{T}_{\varepsilon}$ (inertial manifold) provided that the Lipschitz constant of $F_{\varepsilon}$ is sufficiently small. This manifold can be constructed as a Lipschitz continuous graph over the Banach space $X$, i.e. $\mathfrak{R}_{\varepsilon}=\left\{\left(U, \Phi_{\varepsilon}(U)\right), U \in X\right\}$ (see [2]). From the results due to Chow and Lu [3] it follows that $\mathfrak{M}_{\varepsilon}$ is a $C^{k}$ manifold whenever $F$ and $G$ are $C^{k}$ bounded functions. Notice that, in contrast to the usual definition of an inertial manifold (see, e.g. [4]), we allow $\mathfrak{T}_{\varepsilon}$ to be an infinite dimensional Banach manifold.

In the geometric singular perturbation theory much effort is being spent in order to justify the continuity of the singular limit $\varepsilon$ tends to $0^{+}$(see, e.g. Sviridyuk and Sukacheva [5]). The purpose of this paper is to examine the smoothness of the singular limit behavior of inertial manifolds $\mathfrak{T}_{\varepsilon}$ as $\varepsilon \rightarrow 0^{+}$. The main goal is to show that, for small values of $\varepsilon>0$, the inertial manifold $\mathfrak{T}_{\varepsilon}$ is $C^{1}$ close to the manifold $\mathfrak{T}_{0}=\left\{(U, S), A S=F_{0}(U, S)\right\}$ corresponding to the quasi-dynamic problem $U_{t}=G_{0}(U, S)$ with the constraint $A S=F_{0}(U, S)$. Notice that the $C^{1}$ stability of inertial or centre unstable manifolds is a useful tool in the theory of Morse-Smale vector fields (cf. Mora and Solà-Morales [6]). We hope that $C^{1}$ stability result can be also applied in the theory of linearization at a steady state like, e.g. extension of the Hartman-Grobman lemma from the reduced problem, $\varepsilon=0$ to the perturbed system with $\varepsilon>0$ small enough. Neverthless, such applications of the results obtained are not discussed here.

The idea of construction of an inertial manifold for (1.1) is based on the well-known Lyapunov-Perron method of integral equations. This method is combined with a nonlocal approach using the graph transform which is applied to solutions of the singularly perturbed 
equation in (1.1). We then seek an inertial manifold as the union of all solutions of (1.1) growing exponentially at $-\infty$. By contrast to the usual functional space setting (see, e.g. Chow and Lu [3], Foias et al. [4] or Miklavčič [7]) an essential rôle is played by better smoothing properties of integral kernels enabling us to operate with Hölderian spaces of curves instead of usual continuous ones. We first study the singularly perturbed equation $\varepsilon S_{t}+A S=F_{\varepsilon}(U, S)$ and prove that there is a solution operator $S=\phi_{\varepsilon}(U)$ in the space of globally defined solutions. It, however, turns out that the derivative of this mapping becomes continuous at $\varepsilon=0$ only when the mapping $\phi_{\varepsilon}$ operates on the space of Hölder continuous curves growing exponentially at $-\infty$ (see lemma 3.2). To construct an attractive invariant manifold $\mathfrak{K}_{\varepsilon}$ as a $C^{1}$ graph of $\Phi_{\varepsilon}: X \rightarrow Y^{\alpha}$ we then apply the method of integral equations to the equation $U_{t}=G_{\varepsilon}\left(U, \phi_{\varepsilon}(U)\right)$. In order to prove that $\mathfrak{T}_{\varepsilon}$ is $C^{1}$ close to $\mathfrak{M}_{0}$ for $0<\varepsilon \ll 1$ we make use of the two parameter contraction principle due to Mora and Solà-Morales [6, theorem 5.1] covering differentiability and continuity of a family of nonlinear mappings operating between a pair of Banach spaces.

(1.1) is a semilinear equation $U_{t}+B U=G(U, S)$ and the nonlinearity $F$ only depends on the $U$-variable. The last assumption makes the analysis of the singularly perturbed equation considerably easier. The results obtained in [8] are not capable to cover some applied problems like, e.g. a flow of viscous media governed by a constitutive equation of differential type. Such an application is discussed in Section 4 of this paper.

The paper is organized as follows. In Section 2 we recall some useful results regarding properties of functional spaces of Hölder continuous curves growing exponentially at $-\infty$. In Section 3 we prove that $\Phi_{\varepsilon} \rightarrow \Phi_{0}$ in the $C^{1}$ topology as $\varepsilon \rightarrow 0^{+}$. The main result of this paper is contained in theorem 3.9. Section 4 is devoted to an application of the results obtained to a singular perturbation problem arising in the study of the so-called Johnson-SegalmanOldroyd model of shearing motions of a non-Newtonian fluid. Following the paper by Malkus et al. [9] the motions of the channel Poisseule flow of a highly elastic and very viscous fluid (like, e.g. a polymer) can be described, in a satisfactory manner, by a system of parabolichyperbolic equations of the form

$$
\begin{aligned}
\varepsilon v_{t}-v_{x x} & =\sigma_{x}+f \\
\sigma_{t} & =-\sigma+(1+z) v_{x} \\
z_{t} & =-z-\sigma v_{x},
\end{aligned}
$$

where $v=v(t, x), x \in[0,1]$, is the velocity of the channel flow between two parallel plates, $\sigma$ is the extra shear stress, $z$ is the difference of normal stresses, $f$ is the pressure gradient driving the flow. The number $\varepsilon>0$ is proportional to the ratio of the Reynolds number and Deborah number and according to rheological experiments due to Vinogradov et al. [10] this number is very small, of the order of magnitude $O\left(10^{-12}\right)$. It gives rise to the inertialess approximation $\varepsilon=0$. Based on such an approximation, Malkus et al. [9] were able to explain several striking phenomena like spurt, hysteresis, shape memory and latency observed in rheological experiments (see also [11,12]). Using the new variable $S:=v_{x}+\sigma+f x, S$ is the total stress tensor, system (1.2) can be rewritten in the general form (1.1) with $F_{\varepsilon}=O(\varepsilon)$ as $\varepsilon \rightarrow 0^{+}$. In [13], Nohel and Pego have justified the inertialess approximation by a clever application of the Morse-Conley theory. They proved that any solution of (1.2) converges pointwise for $x \in[0,1]$ 
to a solution of the inertialess approximation as $\varepsilon \rightarrow 0^{+}$. The purpose of Section 4 is to give another justification of the inertialess approximation by means of $C^{1}$ closeness of infinite dimensional inertial manifolds. It is hoped that the $C^{1}$ stability result of inertial manifolds can be also applied to the problem of a piston driven flow studied recently by Malkus et al. [11]. Based on careful numerical simulations, their results indicate the Hopf bifurcation phenomenon in a piston driven Johnson-Segalman-Oldroyd fluid. Any information about $C^{1}$ stability can be a useful tool in order to prove that the Hopf bifurcation extends to the full system of governing equations with $\varepsilon>0$ sufficiently small.

\section{PRELIMINARIES}

As usual, for Banach spaces $E_{1}, E_{2}$ and $\eta \in(0,1]$ we denote $C_{b d d}^{k}\left(E_{1}, E_{2}\right)$ the Banach space consisting of the mappings $F: E_{1} \rightarrow E_{2}$ which are $k$-times Fréchet differentiable and such that $F, \ldots, D^{k} F$ are bounded and uniformly continuous, the norm being given by $\|F\|_{k}:=$ $\sum_{i=0}^{k} \sup \left|D^{i} F\right| . C_{b d d}^{k+\eta}\left(E_{1}, E_{2}\right)$ will denote the Banach space consisting of the mappings $F \in C_{b d d}^{k}\left(E_{1}, E_{2}\right)$ such that $D^{k} F$ is $\eta$-Hölder continuous, the norm being given by

$$
\|F\|_{k, \eta}:=\|F\|_{k}+\sup _{\substack{x \neq y \\ x, y \in E_{1}}} \frac{\left\|D^{k} F(x)-D^{k} F(y)\right\|}{\|x-y\|^{\eta}} .
$$

Let $X$ be a Banach space and $\mu \in R$. Following the notation of $[3,6,7]$ we denote

$$
C_{\mu}^{-}(x):=\left\{u: C((-\infty, 0], x), \text { and }\|u\|_{C_{\mu}^{-}(x)}:=\operatorname{sup~}_{t \leq 0} \mathrm{e}^{\mu t}\|u(t)\|_{x}<\infty\right\} .
$$

The linear space $C_{\mu}^{-}(x)$ endowed with the norm $\|\cdot\|_{C_{\mu}^{-}(x)}$ is a Banach space. If $\mu \leq v$ then the embedding operator $J_{\mu, \nu}: C_{\mu}^{-}(\mathfrak{X}) \rightarrow C_{\nu}^{-}(\mathfrak{X})$ is continuous and $\left\|J_{\mu, \nu}\right\| \leq 1$.

For any $\rho \in(0,1], a \in(0,1]$ and $\mu \geq 0$, we furthermore denote

$$
C_{\mu, \rho, a}^{-}(X)=\left\{u \in C_{\mu}^{-}(X) ;[u]_{\mu, \rho, a}=\sup _{\substack{t \leq 0 \\ h \in(0, a]}} \frac{\left\|\mathrm{e}^{\mu t} u(t)-\mathrm{c}^{\mu(t-h)} u(t-h)\right\|}{h^{\rho}}<\infty\right\}
$$

Let

$$
\|u\|_{C_{\mu, \rho, a}^{-}}(X):=\|u\|_{C_{\mu}^{-}(X)}+[u]_{\mu, \rho, a} \quad \text { for any } u \in C_{\mu, \rho, a}^{-}(X) .
$$

The space $C_{\mu, \rho, a}^{-}(X)$ endowed with the norm $\|\cdot\|_{C_{\mu, \rho, a}^{-}}$is a Banach space continuously embedded into $C_{\mu}^{-}(\mathscr{X})$ with an embedding constant equal to 1 . Furthermore, the space $C_{\mu, \rho, a}^{-}(X)$ is continuously embedded into $C_{v, \rho, a}^{-}(X)$ for any $0 \leq \mu \leq \nu$ and $\rho \in(0,1]$, its embedding constant being less or equal to $\max \left[1,(\nu-\mu) a^{1-\rho}\right\}$ (see [8]).

Let $E_{1}, E_{2}$ be Banach spaces and $F: E_{1} \rightarrow E_{2}$ be a bounded and Lipschitz continuous mapping, $E_{1}, E_{2}$ be Banach spaces. Denote

$$
\tilde{F}: C_{\mu}\left(E_{1}\right) \rightarrow C_{\mu}\left(E_{2}\right)
$$

a mapping defined as $\tilde{F}(u)(t):=F(u(t))$ for any $t \leq 0$ and $u \in C_{\mu}^{-}\left(E_{1}\right)$. By [6, lemma 5.1], for every $\mu \geq 0$, the mapping $\tilde{F}$ is bounded and Lipschitzian with $\sup |\tilde{F}| \leq \sup |F|$ and $\operatorname{Iin}(F)<\operatorname{Lip}(F)$. If $F: E_{1} \rightarrow E_{2}$ is Fréchet differentiable then $\tilde{F}: C_{\mu}^{-}\left(E_{1}\right) \rightarrow C_{\mu}^{-}\left(E_{2}\right)$ need not be necessarily differentiable. Nevertheless, the following result holds. 
Lemma 2.1 [8, lemma 2.8, 14, lemma 5]. If $F: E_{1} \rightarrow E_{2}$ is Fréchet differentiable with $D F: E_{1} \rightarrow L\left(E_{1}, E_{2}\right)$ bounded and uniformly continuous, then, for every $v>\mu, v>0$, the mapping $\bar{F}: C_{\mu}^{-}\left(E_{1}\right) \rightarrow C_{\nu}^{-}\left(E_{2}\right)\left(\tilde{F}: C_{\mu, \rho, a}^{-}\left(E_{1}\right) \rightarrow C_{\nu}^{-}\left(E_{2}\right)\right)$ is Fréchet differentiable, its derivative being given by $D \tilde{F}(u) h=D F(u(\cdot)) h(\cdot)$ and $D \tilde{F}: C_{\mu}^{-}\left(E_{1}\right) \rightarrow L\left(C_{\mu}^{-}\left(E_{1}\right), C_{\nu}^{-}\left(E_{2}\right)\right)$ $\left(D \tilde{F}: C_{\mu, \rho, a}^{-}\left(E_{1}\right) \rightarrow L\left(C_{\mu, \rho, a}^{-}\left(E_{1}\right), C_{\nu}^{-}\left(E_{2}\right)\right)\right)$ is bounded and uniformly continuous.

Throughout Sections 2 and 3 we adopt the following hypothesis

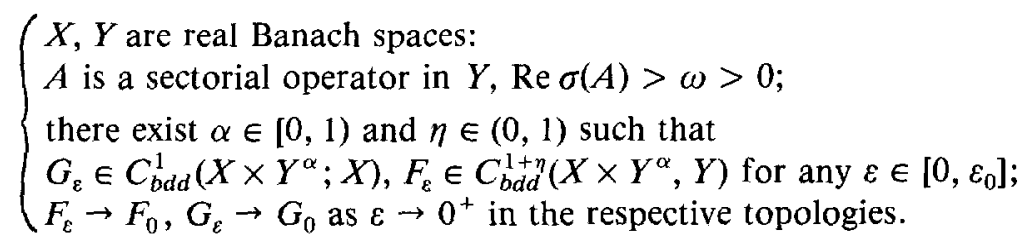

We refer to [1, Chapter 1] for the definition of a sectorial operator, fractional power spaces $Y^{\alpha}, \alpha \geq 0$, and their basic properties. We denote $\|\cdot\|_{\alpha}$ the norm in $Y^{\alpha}$ given by $\|u\|_{\alpha}=\left\|A^{\alpha} u\right\|$, $u \in Y^{\alpha}=D\left(A^{\alpha}\right)$.

By a globally defined solution of (1.1), $\varepsilon>0$, with initial data $\left(U_{0}, S_{0}\right) \in X \times Y^{\alpha}$ we mean a function $(U(\cdot), S(\cdot)) \in C\left([0, T] ; X \times Y^{\alpha}\right) \cap C^{1}\left((0, T) ; X \times Y^{\alpha}\right)$ for any $T>0$ such that $\left.(U(0), S(0))=\left(U_{0}, S_{0}\right) ; U(t), S(t)\right) \in X \times D(A)$ for $t>0$ and $(U(\cdot), S(\cdot))$ satisfies $(1.1)$ for any $t>0$. The global existence and uniqueness of solutions of (1.1), for initial data belonging to the phase-space $X \times Y^{\alpha}$ follow from [1, theorems 3.3.3 and 3.3.4].

In case the function $F_{0}$ satisfies the condition $\left\|D_{S} F_{0}\right\|\left\|A^{\alpha-1}\right\|<1$ the set $\mathfrak{M}_{0}=\{(U, S)$, $\left.A S=F_{0}(U, S)\right\}$ is an embedded Banach manifold in $X \times Y^{\alpha}$. More precisely, there is a $C_{b d d}^{1}$-function $\Phi_{0}: X \rightarrow Y^{\alpha}$ such that

$$
\mathfrak{M}_{0}=\left\{\left(U, \Phi_{0}(U)\right) \in X \times Y^{\alpha}, U \in X\right\} .
$$

By a solution of $(1.1), \varepsilon=0$, we mean a function $U \in C([0, T] ; X) \cap C^{1}((0, T) ; X)$ for any $T>0, U(0)=U_{0}$ and $U(\cdot)$ satisfies the equation $U_{t}=G_{0}\left(U, \Phi_{0}(U)\right)$. Since $G_{0}$ is assumed to be Lipschitz continuous the globai existence and uniqueness of solutions to (1.1) with $\varepsilon=0$ is again assured by the above references to Henry's lecture notes.

In summary, we have shown that the system $(1.1)_{\varepsilon}, \varepsilon>0$ generates a semi-flow $\S_{\varepsilon}(t), t \geq 0$; $S_{\varepsilon}(t)\left(U_{0}, S_{0}\right)=(U(t), S(t))$, on the phase-space $X \times Y^{\alpha}$. The system $(1.1)_{0}$ defines a semi-flow $S_{0}(t), t \geq 0, S_{0}(t)\left(U_{0}, \Phi_{0}\left(U_{0}\right)\right)=\left(U(t), \Phi_{0}(U(t))\right)$, on the embedded manifold $\mathfrak{T}_{0} \subset X \times Y^{\alpha}$.

\section{EXISTENCE AND SMOOTHNESS OF THE SINGULAR LIMIT OF INVARIANT MANIFOLDS}

Before proving the existence and smoothness of the singular limit of inertial manifolds of $(1,1)$ we need several auxiliary lemmas. First, let us examine solutions of the following linear equation

$$
\varepsilon S_{t}+A S=f
$$

belonging to the space $C_{\nu, \rho, a}^{-}\left(Y^{\alpha}\right)$. We will also study the limiting case of $(3.1)_{c}$ when $\varepsilon=0$, i.e.

$$
A S=f
$$

and examine behavior of solutions when $\varepsilon \rightarrow 0^{+}$. 
Denote by $x_{\nu}$ and $x_{\nu, \rho}, v>0,0<\rho \leq 1, a \in(0,1]$ the following Banach spaces of bounded linear operators

$$
\mathfrak{X}_{\nu}=L\left(C_{\nu}^{-}(Y), C_{\nu}^{-}\left(Y^{\alpha}\right)\right), \quad X_{\nu, \rho}=L\left(C_{\nu, \rho, a}^{-}(Y), C_{\nu}^{-}\left(Y^{\alpha}\right)\right) .
$$

Lemma 3.1 [8, lemma 3.1]. Assume that the operator $A$ fulfils the hypothesis (H). Then, for any $\varepsilon \in\left[0, \varepsilon_{0}\right], 0<v<\omega \varepsilon_{0}^{-1}$, and $f \in C_{\nu}^{-}(Y)$ there is the unique solution $S \in C_{\nu}^{-}\left(Y^{\alpha}\right)$ of $(3.1)_{\varepsilon}$ given by $S=L_{\varepsilon} f$, where

$$
L_{\varepsilon} f(t)=\frac{1}{\varepsilon} \int_{-\infty}^{t} \exp (-A(t-s) / \varepsilon) f(s) \mathrm{d} s, \quad(\varepsilon>0) ; \quad L_{0} f(t)=A^{-1} f(t) \quad(\varepsilon=0) .
$$

for $t \leq 0$. The linear operator $L_{\varepsilon}$ belongs to the space $x_{v}$ as well as to $X_{\nu, \rho}, 0<\rho \leq 1$, and there is a $K_{0}>0$ such that $\left\|L_{\varepsilon}\right\|_{x_{v, 0}} \leq\left\|L_{\varepsilon}\right\|_{x_{v}} \leq K_{0}\left(\omega-v \varepsilon_{0}\right)^{\alpha-1}$ for any $\varepsilon \in\left[0, \varepsilon_{0}\right]$, $0<v \varepsilon_{0}<\omega$. Moreover, $L_{\varepsilon} \rightarrow L_{0}$ as $\varepsilon \rightarrow 0^{+}$in the space $x_{\nu, \rho}, 0<\rho \leq 1$.

Lemma 3.2. Let $0<(1+\eta) \mu \leq \kappa<\omega \varepsilon_{0}^{-1}, 0<\rho \leq 1$ and $0<a \leq 1$. Assume that there is a $\theta<1$ such that $\left\|L_{\varepsilon}\right\|_{\mathscr{S}_{\mu}}\left\|D_{S} F_{\varepsilon}(U, S)\right\|_{L\left(Y^{\alpha}, Y\right)} \leq \theta$ for any $U \in X, S \in Y^{\alpha}$ and $\varepsilon \in\left[0, \varepsilon_{0}\right]$. Then, for any $U \in C_{\mu}^{-}(X)$ there is the unique solution $S=\phi_{\varepsilon}(U) \in C_{\mu}^{-}\left(Y^{\alpha}\right)$ of the equation $S=L_{\varepsilon} F_{\varepsilon}(U, S)$. Moreover, there exists a $K_{1}>0$ such that, for any $\varepsilon \in\left[0, \varepsilon_{0}\right]$,

(i) $\left\|\phi_{\varepsilon}\left(U_{1}\right)-\phi_{\varepsilon}\left(U_{2}\right)\right\|_{C_{\mu^{-}\left(Y^{\alpha}\right)}} \leq\left\|L_{\varepsilon}\right\|_{\mathscr{X}_{\mu}}\left\|F_{\varepsilon}\right\|_{1}(1-\theta)^{-1}\left\|U_{1}-U_{2}\right\|_{C_{\mu(X)}^{-}\left(U^{\prime}\right)}$

(ii) $\lim _{\varepsilon \rightarrow 0^{+}} \phi_{\varepsilon}(U)=\phi_{0}(U)$ in $C_{\mu}^{-}\left(Y^{\alpha}\right)$ uniformly w.r. to $U \in B$, where $B$ is an arbitrary bounded subset of $C_{\mu, \rho, a}^{-}(X)$;

(iii) $\phi_{\varepsilon} \in C_{b d d}^{1}\left(C_{\mu}^{-}(X), C_{\kappa}^{-}\left(Y^{\alpha}\right)\right),\left\|\phi_{\varepsilon}\right\|_{1} \leq K_{1}$ and there is a $d \phi_{\varepsilon} \in L\left(C_{\mu}^{-}(X), C_{\mu}^{-}\left(Y^{\alpha}\right)\right)$ with the property $D \phi_{\varepsilon}=J_{\mu, \kappa} d \phi_{\varepsilon},\left\|d \phi_{\varepsilon}\right\| \leq\left\|L_{\varepsilon}\right\|_{X_{\mu}}\left\|F_{\varepsilon}\right\|_{1}(1-\theta)^{-1}$;

(iv) $\lim _{\varepsilon \rightarrow 0^{+}} \phi_{\varepsilon}=\phi_{0}$ in $C_{b d d}^{1}\left(B, C_{\kappa}^{-}\left(Y^{\alpha}\right)\right)$ for any bounded and open subset $\mathbb{B}$ of $C_{\mu, \rho, a}^{-}(X)$.

Remark 3.3. It follows from the proof of $\left[8\right.$, lemma 3.1] that $\left\|I_{\varepsilon}-L_{0}\right\|_{\mathfrak{X}_{\mu, \rho}}=O\left(\varepsilon^{r}\right)$ as $\varepsilon \rightarrow 0^{+}$for any $0<r<1$. The author was able to prove neither $C^{1}$ differentiability nor Lipschitz continuity of $L_{\varepsilon}$ with respect to $\varepsilon$ at $\varepsilon=0$.

Remark 3.4. We remind ourselves that in the case $\varepsilon=0$ the mapping $\Phi_{0}$ defined in (2.1) coincides with $\phi_{0}$ in the sense that $\phi_{0}(U)(t)=\Phi_{0}(U(t))$ for any $U \in C_{\mu}^{-}(X)$ and $t \leq 0$.

Proof of lemma 3.2. Under the assumption $\left\|L_{\varepsilon}\right\|_{\mathfrak{x}_{\mu}}\left\|D_{S} F_{\varepsilon}\right\|_{L\left(Y^{\alpha}, Y\right)} \leq \theta<1$ the existence of the solution operator $S=\phi_{\varepsilon}(U)$ as well as its Lipschitz continuity (i) follows from the parameterized contraction principle.

To prove (ii), we first find an estimate of the norm of $\left\|\phi_{0}(U)\right\|_{C_{\mu, \rho, a}^{-}\left(Y^{\alpha}\right)}$ in terms of $U \in C_{\mu, \rho, a}^{-}(X)$. To this end, we put $S(t)=\phi_{0}(U)(t)$. Then, for any $t \leq 0, h \in(0, a]$, we have

$$
\begin{aligned}
\mathrm{e}^{\mu t} S(t)-\mathrm{e}^{\mu(t-h)} S(t-h)= & \left(\mathrm{e}^{\mu t}-\mathrm{e}^{\mu(t-h)}\right) A^{-1} F_{0}(U(t), S(t)) \\
& +\mathrm{e}^{\mu(t-h)} A^{-1}\left(F_{0}(U(t), S(t))-F_{0}(U(t-h), S(t-h))\right) .
\end{aligned}
$$

Notice that, for any $t \leq 0, h \in(0, a]$,

$$
\begin{aligned}
\|W(t)-W(t-h)\|_{E} & \leq \mathrm{e}^{-\mu t}\left\|\mathrm{e}^{\mu t} W(t)-\mathrm{e}^{\mu(t-h)} W(t-h)\right\|_{E}+\left(1-\mathrm{e}^{-\mu h}\right)\|W(t-h)\|_{E} \\
& \leq K_{1} \mathrm{e}^{-\mu t}\|W\|_{C_{\mu, \rho, a}^{-}(E)} h^{\rho}
\end{aligned}
$$


where $E$ stands either for $X$ or $Y^{\alpha}$ and $K_{1}=K_{1}(\mu)>0$. Thus,

$$
\begin{aligned}
\left\|\mathrm{e}^{\mu t} S(t)-\mathrm{e}^{\mu(t-h)} S(t-h)\right\|_{Y^{\alpha}} \leq & K_{1}\|U\|_{\mathcal{C}_{\mu, \rho, \alpha}^{-}(X)} h^{\rho} \\
& +\left\|A^{\alpha-1}\right\|\left\|D_{S} F_{0}\right\|\|S(t)-S(t-h)\|_{Y^{\alpha}} \mathrm{e}^{\mu(t-h)} .
\end{aligned}
$$

Since $\|S\|_{C_{\mu}^{-}\left(Y^{\alpha}\right)} \leq\left\|A^{\alpha-1}\right\|\left\|F_{0}\right\|_{0}$ and $\left\|L_{0}\right\|_{\mathfrak{x}_{\mu}}\left\|D_{S} F_{0}\right\| \leq \theta<1$ the above inequality yields the estimate

$$
\left\|\phi_{0}(U)\right\|_{C_{\beta, p, a}^{-}\left(Y^{\alpha}\right)} \leq K_{1}\left(1+\|U\|_{C_{\beta, p, a}^{-}(X)}\right)
$$

Arguing similarly as above one can show $\left\|F_{0}(U, S)\right\|_{C_{\mu, \rho, u}^{-}(Y)} \leq K_{1}\left(1+\|U\|_{C_{\mu, \rho, a}^{-}(X)}+\right.$ $\left.\|S\|_{C_{\mu, \rho, a}^{-}\left(Y^{\alpha}\right)}\right)$. Hence,

$$
\left\|F_{0}\left(U, \phi_{0}(U)\right)\right\|_{C_{\mu, \rho, \alpha}^{-}(Y)} \leq K_{1}\left(1+\|U\|_{C_{\mu, \rho, \alpha}^{-}(X)}\right) .
$$

As $\phi_{\varepsilon}(U)=L_{\varepsilon} F_{\varepsilon}\left(U, \phi_{\varepsilon}(U)\right)$ we obtain

$$
\begin{aligned}
(1-\theta)\left\|\phi_{\varepsilon}(U)-\phi_{0}(U)\right\|_{C_{\mu}^{-}\left(Y^{\alpha}\right)} \leq & \left\|L_{\varepsilon}-L_{0}\right\|_{X_{\mu, \rho}}\left\|F_{0}\left(U, \phi_{0}(U)\right)\right\|_{C_{\mu, \rho, q}^{-}(Y)} \\
& +\left\|L_{\varepsilon}\right\|_{X_{\mu}}\left\|F_{\varepsilon}\left(U, \phi_{0}(U)\right)-F_{0}\left(U, \phi_{0}(U)\right)\right\|_{C_{\mu}^{-}(Y)} .
\end{aligned}
$$

By lemma 3.1, (H) and (3.5) we obtain $\lim _{\varepsilon \rightarrow 0^{+}} \phi_{\varepsilon}(U)=\phi_{0}(U)$ in $C_{\mu}^{-}\left(Y^{\alpha}\right)$ uniformly w.r. to $U \in \mathbb{B}$ where $B$ is arbitrary bounded subset of $C_{\mu, \rho, a}^{-}(X)$.

(iii) For any $U, W \in C_{\mu}^{-}(X)$ we denote

$$
D \phi_{\varepsilon}(U) W:=\left[I-L_{\varepsilon} D_{S} F_{\varepsilon}\left(U(\cdot), \phi_{\varepsilon}(U)(\cdot)\right)\right]^{-1} L_{\varepsilon} D_{U} F_{\varepsilon}\left(U(\cdot), \phi_{\varepsilon}(U)(\cdot)\right) W .
$$

A straightforward calculation yields

$$
\begin{aligned}
\phi_{\varepsilon}(U+W)-\phi_{\varepsilon}(U)-D \phi_{\varepsilon}(U) W= & B_{\varepsilon}\left[F_{\varepsilon}\left(U+W, \phi_{\varepsilon}(U)\right)-F_{\varepsilon}\left(U, \phi_{\varepsilon}(U)\right)-D_{U} F_{\varepsilon}\left(U, \phi_{\varepsilon}(U)\right) W\right] \\
& +B_{\varepsilon}\left[F_{\varepsilon}\left(U+W, \phi_{\varepsilon}(U+W)\right)-F_{\varepsilon}\left(U+W, \phi_{\varepsilon}(U)\right)\right. \\
& \left.-D_{S} F_{\varepsilon}\left(U, \phi_{\varepsilon}(U)\right)\left(\phi_{\varepsilon}(U+W)-\phi_{\varepsilon}(U)\right)\right]=: I_{1}+I_{2},
\end{aligned}
$$

where

$$
B_{\varepsilon}:=\left[I-L_{\varepsilon} D_{S} F_{\varepsilon}\left(U(\cdot), \phi_{\varepsilon}(U)(\cdot)\right)\right]^{-1} L_{\varepsilon} .
$$

Obviously, $\left\|B_{\varepsilon}\right\|_{\mathfrak{X}_{\nu}} \leq(1-\theta)^{-1}\left\|L_{\varepsilon}\right\|_{\mathfrak{X}_{\nu}}$ for $v=\mu$ or $v=\kappa, \varepsilon \in\left[0, \varepsilon_{0}\right]$. Furthermore, by lemma 2.1 , we have $\left\|I_{1}\right\|_{C_{\kappa}^{-}\left(Y^{\alpha}\right)}=o\left(\|W\|_{C_{\mu}^{-}(X)}\right)$ as $\|W\| \rightarrow 0$. On the other hand, as $F_{\varepsilon} \in C_{b d d}^{1+\eta}$ and $0<(1+\eta) \mu \leq \kappa$ we have

$$
\begin{aligned}
\left\|I_{2}\right\|_{C_{\kappa}^{-}\left(Y^{\alpha}\right)} & =\mathrm{O}\left(\|W\|_{C_{\mu}^{-}}^{\eta}+\left\|\phi_{\varepsilon}(U+W)-\phi_{\varepsilon}(U)\right\|_{C_{\mu}^{-}}^{\eta}\right)\left\|\phi_{\varepsilon}(U+W)-\phi_{\varepsilon}(U)\right\|_{C_{\mu}^{-}} \\
& =o\left(\|W\|_{C_{\nu}^{-}}\right) .
\end{aligned}
$$

Hence, $\phi_{\varepsilon} \in C_{b d d}^{1}\left(C_{\mu}^{-}(X), C_{\kappa}^{-}\left(Y^{\alpha}\right)\right) ; D \phi_{\varepsilon}(U) W=J_{\mu, \kappa} d \phi_{\varepsilon}(U) W$, where the mapping $W \mapsto$ $d \phi_{\varepsilon}(U) W$ is defined by the right-hand side of (3.6) and so $\left\|d \phi_{\varepsilon}\right\| \leq\left\|L_{\varepsilon}\right\|_{\chi_{\mu}}\left\|F_{\varepsilon}\right\|_{1}(1-\theta)^{-1}$. 
Finally, we prove the assertion (iv). Let $B \subset C_{\mu, \rho, a}^{-}(X)$ be an arbitrary bounded set. With regard to (ii) it is sufficient to show the uniform convergence $D \phi_{\varepsilon}(U) \rightarrow D \phi_{0}(U)$ as $\varepsilon \rightarrow 0^{+}$for $U \in \Re$. For any $U \in C_{\mu, \rho, a}^{-}(X)$ we have

$$
\begin{aligned}
D \phi_{\varepsilon}(U)-D \phi_{0}(U)= & \left(B_{\varepsilon}-B_{0}\right) D_{U} F_{0}\left(U, \phi_{0}(U)\right) \\
& +B_{\varepsilon}\left[D_{U} F_{\varepsilon}\left(U, \phi_{\varepsilon}(U)-D_{U} F_{0}\left(U, \phi_{0}(U)\right)\right] .\right.
\end{aligned}
$$

Now one can readily verify that

$$
\begin{aligned}
B_{\varepsilon}-B_{0}= & B_{\varepsilon}\left[D_{S} F_{\varepsilon}\left(U, \phi_{\varepsilon}(U)\right)-D_{S} F_{0}\left(U, \phi_{0}(U)\right)\right] B_{0} \\
& +\left[I-L_{\varepsilon} D_{S} F_{\varepsilon}\left(U, \phi_{\varepsilon}(U)\right)\right]^{-1}\left(L_{\varepsilon}-L_{0}\right)\left(I+D_{S} F_{0}\left(U, \phi_{0}(U)\right) B_{0}\right) .
\end{aligned}
$$

Furthermore,

$$
\begin{aligned}
D_{S} F_{\varepsilon}\left(U, \phi_{\varepsilon}(U)\right)-D_{S} F_{0}\left(U, \phi_{0}(U)\right)= & D_{S}\left[F_{\varepsilon}\left(U, \phi_{\varepsilon}(U)\right)-F_{0}\left(U, \phi_{\varepsilon}(U)\right)\right] \\
& +D_{S}\left[F_{0}\left(U, \phi_{\varepsilon}(U)\right)-\Gamma_{0}\left(U, \phi_{0}(U)\right)\right] .
\end{aligned}
$$

Thus,

$$
\begin{aligned}
& \left\|D_{S} F_{\varepsilon}\left(U(t), \phi_{\varepsilon}(U)(t)\right)-D_{S} F_{0}\left(U(t), \phi_{0}(U)(t)\right)\right\|_{L\left(Y^{\alpha}, Y\right)} \\
& \quad \leq\left\|F_{\varepsilon}-F_{0}\right\|_{1}+\left\|F_{0}\right\|_{1+\eta}\left\|\phi_{\varepsilon}(U)(t)-\phi_{0}(U)(t)\right\|_{Y^{\alpha}}^{n} .
\end{aligned}
$$

Since $0<(1+\eta) \mu \leq \kappa$, we obtain

$$
\begin{aligned}
& \left\|D_{S} F_{\varepsilon}\left(U, \phi_{\varepsilon}(U)\right)-D_{S} F_{0}\left(U, \phi_{0}(U)\right)\right\|_{L\left(C_{\mu}^{-}\left(Y^{\alpha}\right), C_{x}^{-}(Y)\right)} \\
& \quad \leq\left\|F_{\varepsilon}-F_{0}\right\|_{1}+\left\|F_{0}\right\|_{1+\eta}\left\|\phi_{\varepsilon}(U)-\phi_{0}(U)\right\|_{C_{\mu}^{-}\left(Y^{\alpha}\right)}^{\eta} .
\end{aligned}
$$

However, the right-hand side of the above inequality tends to 0 as $\varepsilon \rightarrow 0^{+}$uniformly w.r. to $u \in \mathbb{B}$. Similarly, one has

$$
\left\|D_{U} F_{\varepsilon}\left(U, \phi_{\varepsilon}(U)\right)-D_{U} F_{0}\left(U, \phi_{0}(U)\right)\right\|_{L\left(C_{\mu}^{-}(X), C_{x}^{-}(Y)\right)} \rightarrow 0 \quad \text { as } \varepsilon \rightarrow 0^{+}
$$

uniformly w.r. to $U \in \mathbb{B}$. Now we notice that $\left\|B_{0} D_{U} F_{0}\left(U, \phi_{0}(U)\right)\right\|_{L\left(C_{\mu}^{-}(X), C_{\mu}^{-}\left(Y^{\alpha}\right)\right)} \leq K_{1}$ and

$$
\left\|\left[I+D_{S} F_{0}\left(U, \phi_{0}(U)\right) B_{0}\right] D_{U} F_{0}\left(U, \phi_{0}(U)\right)\right\|_{L\left(C_{\mu, \rho, a}^{-}(X), C_{\mu, \rho, \alpha}^{-}(Y)\right)} \leq K_{1}\left(1+\|U\|_{C_{\mu, \rho, Q}^{-}(X)}^{\eta}\right) .
$$

Indeed, let us denote

$$
A(t):=\left[I+D_{S} F_{0}\left(U(t), \phi_{0}(U)(t)\right) B_{0}\right] D_{U} F_{0}\left(U(t), \phi_{0}(U)(t)\right), \quad t \leq 0 .
$$

Then, by (3.3) and (3.4),

$$
\begin{aligned}
\|A(t)-A(t-h)\|_{L(X, Y)} & \leq K_{1}\left(\|U(t)-U(t-h)\|_{X}^{\eta}+\left\|\phi_{0}(U)(t)-\phi_{0}(U)(t-h)\right\|_{Y^{\alpha}}^{\eta}\right) \\
& \leq K_{1} \mathrm{e}^{-\mu \eta t} h^{\eta \rho}\left(1+\|U\|_{C_{\mu, \rho, Q}^{-}(X)}^{\eta}\right) .
\end{aligned}
$$

As $0<(1+\eta) \mu \leq \kappa$ we oblain $\|A(\cdot) W\|_{C_{\kappa, \nu p, a}^{-}(Y)} \leq K_{1}\|W\|_{C_{\mu, \rho, a}^{-}(X)}\left(1+\|U\|_{C_{\mu, o, a}^{-}(X)}^{\eta^{-}}\right)$for any $W \in C_{\mu, \rho, a}^{-}(X)$. According to lemma 3.2 it is now obvious that $D \phi_{\varepsilon}(U) \rightarrow D \phi_{0}(U)$ as $\varepsilon \rightarrow 0^{+}$ uniformly w.r. to $U \in \mathbb{B}$. The proof of lemma 3.2 is complete.

We will construct an inertial manifold $\mathfrak{N}_{\varepsilon}$ for the semi-flow $S_{\varepsilon}$ as the union of all Hölder continuous curves growing exponentially at $-\infty$, i.e.

$$
\mathfrak{K}_{\varepsilon}=\left\{\left(Y(\tau), \tau \in R, Y \in C_{\mu, \rho, a}^{-}\left(X \times Y^{\alpha}\right),(U(\cdot), S(\cdot))=Y(\cdot) \text { solves }(1.1)\right\}\right.
$$


for some $\mu>0, \rho \in(0,1)$ and $a \in(0,1]$. The invariance property of $\mathfrak{T}_{\varepsilon}$ under the semiflow $S_{\varepsilon}(t), t \geq 0$, generated by system (1.1) is obvious. According to lemmas 3.1 and 3.2 $(U(\cdot), S(\cdot)) \in C_{\mu, \rho, a}^{-}\left(X \times Y^{\alpha}\right)$ is a solution of (1.1) if and only if it satisfies the following integral equation

$$
U(t)=x+\int_{0}^{t} G_{\varepsilon}\left(U(s), \phi_{\varepsilon}(U)(s)\right) \mathrm{d} s=: T_{\varepsilon}(x, U)(t) \quad \text { for any } t \leq 0
$$

for some $x \in X$. Using the invariance property of $\mathfrak{N}_{\varepsilon}$ we can write the set $\mathfrak{M}_{\varepsilon}$ as

$$
\mathfrak{M}_{\varepsilon}=\left\{\left(x, \phi_{\varepsilon}(U)(0)\right), x \in X, U=T_{\varepsilon}(x, U) \in C_{\mu, \rho, a}^{-}(X)\right\} .
$$

In what follows we will investigate the existence and the limiting behavior of fixed points of the two parameter family of mappings

$$
T_{\varepsilon}(x, \cdot): C_{\mu, \rho, a}^{-}(X) \rightarrow C_{\mu, \rho, a}^{-}(X), \quad \varepsilon \in\left[0, \varepsilon_{0}\right], x \in X,
$$

defined by the right-hand side of (3.8). We are going to prove that $T_{\varepsilon}(x, \cdot)$ is a uniform contraction. If $\left\|L_{\varepsilon}\right\|_{\mathfrak{x}_{n}}\left\|D_{S} F_{\varepsilon}\right\| \leq \theta<1$ then by lemma 3.2(i), we have

$$
\begin{aligned}
& \left\|G_{\varepsilon}\left(U_{1}, \phi_{\varepsilon}\left(U_{1}\right)\right)-G_{\varepsilon}\left(U_{2}, \phi_{\varepsilon}\left(U_{2}\right)\right)\right\|_{C_{\mu}^{-(}(X)} \\
& \quad \leq\left\|G_{\varepsilon}\right\|_{1}\left(1+K_{0}\left\|F_{\varepsilon}\right\|_{1}\left(\omega-\mu \varepsilon_{0}\right)^{\alpha-1}(1-\theta)^{-1}\right)\left\|U_{1}-U_{2}\right\|_{C_{\mu}^{-}(X)},
\end{aligned}
$$

where $K_{0}>0$ is a constant independent of $0<\mu<\omega \varepsilon_{0}^{-1}$. Assume that $0<\rho<1$ and $v>0$. Then the linear operator

$$
J: g \mapsto \int_{0}^{t} g(s) \mathrm{d} s, \quad J: C_{\nu}^{-}(X) \rightarrow C_{\nu, \rho, a}^{-}(X)
$$

is bounded its norm being estimated by

$$
\|J\|_{L\left(C_{v}^{-}(X), C_{\nu, \beta, a}^{-}(X)\right)} \leq \frac{2}{v}
$$

provided that $a=a(v)>0$ sufficiently small (c.f. [8, lemma 3.2,c]).

By the next lemma 3.5 we will show that under an additional assumption on $D_{S} F_{\varepsilon}$ the following hypotheses are fulfilled

(T)

(1) there is $\theta<1$ with the property $\left\|T_{\varepsilon}\left(x, U_{1}\right)-T_{\varepsilon}\left(x, U_{2}\right)\right\|_{\mathcal{U}} \leq \theta\left\|U_{1}-U_{2}\right\|_{\mathcal{U}}$ for any $x \in \mathscr{X}, U_{1}, U_{2} \in \mathcal{U}$ and $\varepsilon \in\left[0, \varepsilon_{0}\right]$;

(2) there is a $Q<\infty$ such that $\left\|T_{\varepsilon}\left(x_{1}, U\right)-T_{\varepsilon}\left(x_{2}, U\right)\right\|_{\mathcal{U}} \leq Q\left\|x_{1}-x_{2}\right\|_{X}$ for any $x_{1}, x_{2} \in X, U \in \mathcal{U}$ and $\varepsilon \in\left[0, \varepsilon_{0}\right]$

(3) for any bounded open subset $B \subset X$,

$$
\sup _{x \in B}\left\|T_{\varepsilon}\left(x, U_{0}(x)\right)-T_{0}\left(x, U_{0}(x)\right)\right\|_{u} \rightarrow 0 \varepsilon \rightarrow 0^{+}
$$

where $U_{\varepsilon}(x), x \in X, \varepsilon \in\left[0, \varepsilon_{0}\right]$, is the unique fixed point of $T_{\varepsilon}(x, U)=U$ in $\mathcal{U}$

on the Banach spaces

$$
\mathcal{U}:=C_{\mu, \rho, a}^{-}(X), \quad \bar{u}:=C_{\kappa, \rho, a}^{-}(X), \quad 0<(1+\eta) \mu<\kappa<\omega \varepsilon_{0}^{-1},
$$

where $0<\rho<1$ is fixed and $a \in(0,1]$ is such that the estimate (3.12) holds for both values $v=\mu$ as well as $v=\kappa$. 
LEMMA 3.5. Assume that the hypotheses $(\mathrm{H})$ are fulfilled. Then there is a positive number $\delta>0$ such that if $\left\|D_{S} F_{\varepsilon}\right\|_{L\left(Y^{\alpha}, Y\right)} \leq \delta$ for any $\varepsilon \in\left[0, \varepsilon_{0}\right]$ then there exists an invariant manifold $\mathfrak{T}_{\varepsilon}$, $\varepsilon \in\left[0, \varepsilon_{0}\right]$, for the semi-flow $S_{\varepsilon}$ generated by the system of equations (1.1). This manifold is a graph, $\mathfrak{T}_{\varepsilon}=\left\{\left(x, \Phi_{\varepsilon}(x)\right), x \in X\right\}$, where $\Phi_{\varepsilon}: X \rightarrow Y^{\alpha}$ is a bounded Lipschitz continuous function. Moreover, for any bounded subset $B \subset X, \lim _{\varepsilon \rightarrow 0^{+}} \Phi_{\varepsilon}(x)=\Phi_{0}(x)$ uniformly w.r. to $x \in B$.

If, in addition, the operator $A$ has a compact resolvent $A^{-1}: Y \rightarrow Y$ then the manifold $\mathfrak{K}_{\varepsilon}$ is also exponentially attractive, i.e. there is a $\mu>0$ such that $\operatorname{dist}\left((U(t), S(t)), \mathfrak{T}_{\varepsilon}\right)=O\left(\mathrm{e}^{-\mu t}\right)$ when $t \rightarrow \infty$ for any solution $(U(\cdot), S(\cdot))$ of $(1.1), \varepsilon \in\left(0, \varepsilon_{0}\right]$.

Proof. According to lemma 3.2, for any $\mu>0$, we can choose an $\varepsilon_{0}=\varepsilon(\mu) \ll 1$ such that $\left\|L_{\varepsilon}\right\|_{\mathscr{X}_{\mu}} \leq K_{0}\left(\omega-\mu \varepsilon_{0}\right)^{\alpha-1} \leq K_{0}(\omega / 2)^{\alpha-1}$ for any $\varepsilon \in\left[0, \varepsilon_{0}(\mu)\right]$. Let $0<\delta \ll 1$ be such that $K_{0}(\omega / 2)^{\alpha-1} \delta<1$. Now, if we suppose $\left\|D_{S} F_{\varepsilon}\right\| \leq \delta, \varepsilon \in\left[0, \varepsilon_{0}\right]$, we obtain the estimate (3.11) for the Lipschitz constant of the mapping $C_{\mu}^{-}(X) \ni U \mapsto G_{\varepsilon}\left(U, \phi_{\varepsilon}(U)\right) \in C_{\mu}^{-}(X)$ with some $\theta=K_{0}(\omega / 2)^{\alpha-1} \delta<1$. With regard to (3.12) one can furthermore choose $\mu \gg 1$ large enough and such that the mapping $T_{\varepsilon}(x, \cdot): \mathcal{U} \rightarrow \mathcal{U}$ fulfils the hypothesis $(T)_{1}$. The Lipschitz constant $Q$ of the mapping $x \mapsto T_{\varepsilon}(x, U)$ is equal to 1 . Let $U_{0}=U_{0}(x)$ be the unique fixed point of $U_{0}=T_{0}\left(x, U_{0}\right)$. Then, for any bounded and open subset $B \subset X$, we have $\left\|U_{0}(x)\right\|_{\mathcal{U}} \leq\|x\|_{X}+$ $\|J\|_{L\left(C_{\mu}^{-}(X), \text { u }\right)}\left\|G_{0}\right\|_{0} \leq K_{0}(B)$ for every $x \in B$. Moreover,

$$
\begin{aligned}
\left\|T_{\varepsilon}\left(x, U_{0}(x)\right)-T_{0}\left(x, U_{0}(x)\right)\right\|_{u} & \leq\|\Im\|\left\|G_{\varepsilon}\left(U_{0}, \phi_{\varepsilon}\left(U_{0}\right)\right)-G_{0}\left(U_{0}, \phi_{0}\left(U_{0}\right)\right)\right\|_{C_{\mu}^{-}(X)} \\
& \leq(2 / \mu)\left\|G_{\varepsilon}\right\|_{1}\left\|\phi_{\varepsilon}\left(U_{0}\right)-\phi_{0}\left(U_{0}\right)\right\|_{C_{\mu}^{-}(X)}+O\left(\left\|G_{\varepsilon}-G_{0}\right\|\right) .
\end{aligned}
$$

Due to lemma 3.3(ii), we know that $\left\|\phi_{\varepsilon}\left(U_{0}(x)\right)-\phi_{0}\left(U_{0}(x)\right)\right\|_{C_{\mu}^{-}\left(Y^{\alpha}\right)} \rightarrow 0$ as $\varepsilon \rightarrow 0^{+}$uniformly w.r. to $x \in B$ and so the hypothesis (T) $)_{3}$ is also satisfied.

Define $\Phi_{\varepsilon}(x):=\phi_{\varepsilon}\left(U_{\varepsilon}(x)\right)(0)$. According to (3.9) we have that the set $\mathfrak{T}_{\varepsilon}$ is a graph over the Banach space $X$, i.e. $\mathfrak{T}_{\varepsilon}=\left\{\left(x, \Phi_{\varepsilon}(x)\right), x \in X\right\}$ and moreover, as the mapping $x \mapsto U_{\varepsilon}(x)$ and $\phi_{\varepsilon}$ are Lipschitz continuous, $\Phi_{\varepsilon}$ is Lipschitz continuous as well. Hence, $\mathfrak{M}_{\varepsilon}$ is an invariant Lipschitz manifold for the semi-flow $S_{\varepsilon}$ generated by $(1.1), \varepsilon \in\left(0, \varepsilon_{0}\right]$. Since $\left\|L_{0}\right\|_{\mathscr{X}_{\mu}}=\left\|A^{\alpha-1}\right\|$ we have $\left\|A^{\alpha-1}\right\|\left\|D_{S} F_{0}\right\| \leq \theta<1$ and so, by definition of a solution of $(1.1), \varepsilon=0$, the set $\mathfrak{M}_{0}$ defined by (2.1) is an invariant manifold for the semi-flow $S_{0}$. With regard to remark 3.4, we again have $\Phi_{0}(x)=\phi_{0}\left(U_{0}(x)\right)(0)$.

Let $B \subset X$ be a bounded subset. From $(\mathrm{T})_{1}$ and $(\mathrm{T})_{3}$ it follows that $U_{\varepsilon}(x) \rightarrow U_{0}(x)$ as $\varepsilon \rightarrow 0^{+}$ uniformly w.r. to $x \in B$. Then by lemma 3.2(i),(ii), we have $\Phi_{\varepsilon}(x) \rightarrow \Phi_{0}(x)$ in $Y^{\alpha}$ as $\varepsilon \rightarrow 0^{+}$ uniformly w.r. to $x \in B$.

The proof of exponential attractivity of $\mathfrak{T}_{\varepsilon}, \varepsilon \in\left(0, \varepsilon_{0}\right]$ is similar, in spirit, to that of the paper by Chow and $\mathrm{Lu}$ [3, theorem 5.1]. In fact, it follows the lines of known proofs of existence of stable invariant foliation to a centre-unstable manifold. Let $\varepsilon \in\left(0, \varepsilon_{0}\right]$ be fixed. Given a solution $(\bar{U}, \bar{S})$ of $(1.1)$ we want to find a solution $\left(U^{*}, S^{*}\right) \in \mathfrak{T}_{\varepsilon}$ with the property $(U, S) \in$ $C_{\mu}^{+}\left(X \times Y^{\alpha}\right)$ for some $\mu>0$ where $U=U^{*}-\bar{U}, S=S^{*}-\bar{S}$ and $C_{\mu}^{+}$is the Banach space

$$
C_{\mu}^{+}\left(X \times Y^{\alpha}\right):=\left\{f \in C\left(R^{+}, X \times Y^{\alpha}\right),\|f\|_{C_{\mu}^{+}}=\sup _{t \geq 0} \mathrm{e}^{\mu t}\|f(t)\|_{X \times Y^{\alpha}}<\infty\right\} .
$$

Obviously, the existence of such a solution would imply that the $\mathfrak{T}_{\varepsilon}$ manifold has the exponential tracking property and as a consequence we would have $\operatorname{dist}\left((U(t), S(t)), \mathfrak{T}_{\varepsilon}\right)=O\left(\mathrm{e}^{-\mu t}\right)$ when $t \rightarrow \infty$, i.e. $\mathfrak{K}_{\varepsilon}$ is an exponentially attractive invariant manifold. 
Now, one easily verifies that $(U, S)$ belongs to $C_{\mu}^{+}, \mu>\left\|G_{\varepsilon}\right\|_{1}$, if and only if the following integral equations are satisfied

$$
\begin{aligned}
U(t)= & \int_{\infty}^{t} G_{\varepsilon}(\bar{U}(s)+U(s), \bar{S}(s)+S(s))-G_{\varepsilon}(\bar{U}(s), \bar{S}(s)) \mathrm{d} s=: \mathcal{F}^{U}(U, S)(t) \\
S(t)= & \exp (-A t / \varepsilon) \xi+\frac{1}{\varepsilon} \int_{0}^{t} \exp (-A(t-s) / \varepsilon)\left[F_{\varepsilon}(\bar{U}(s)+U(s), \bar{S}(s)+S(s))\right. \\
& \left.-F_{\varepsilon}(\bar{U}(s), \bar{S}(s))\right] \mathrm{d} s \quad \text { for any } t \geq 0
\end{aligned}
$$

for some $\xi \in Y^{\alpha}$. The operator $\mathcal{F}^{U}$ defined by the right-hand side of the first equation in (3.14) is well posed on the space $C_{\mu}^{+}\left(X \times Y^{\alpha}\right)$ with values in $C_{\mu}^{+}(X)$. Moreover, the mapping $U \mapsto \mathcal{F}^{U}(U, S)$ is a uniform contraction in $C_{\mu}^{+}(X)$ provided that $\mu>\left\|G_{\varepsilon}\right\|_{1}$. More precisely, one has

$$
\left\|\mathscr{F}^{U}\left(U_{1}, S\right)-\mathcal{F}^{U}\left(U_{2}, S\right)\right\|_{C_{\mu}^{-1}(X)} \leq\left\|G_{\varepsilon}\right\|_{1} \mu^{-1}\left\|U_{1}-U_{2}\right\|_{C_{\mu}^{\prime}(X)}
$$

and, similarly,

$$
\left\|\mathfrak{F}^{U}\left(U, S_{1}\right)-\mathfrak{F}^{U}\left(U, S_{2}\right)\right\|_{C_{\mu}^{+}(X)} \leq\left\|G_{\varepsilon}\right\|_{1} \mu^{-1}\left\|S_{1}-S_{2}\right\|_{C_{\mu}^{+}\left(Y^{\alpha}\right)} .
$$

By the parameterized contraction principle there is a mapping $h: C_{\mu}^{+}\left(Y^{\alpha}\right) \rightarrow C_{\mu}^{+}(X)$ such that, for any $S \in C_{\mu}^{+}\left(Y^{\alpha}\right), U \in C_{\mu}^{+}(X)$ is a solution of $U=\mathscr{F}^{U}(U, S)$ iff $U=h(S)$. The Lipshitz constant of the mapping $h$ can be estimated as

$$
\left\|h\left(S_{1}\right)-h\left(S_{2}\right)\right\|_{C_{\mu}^{+}(X)} \leq \frac{\left\|G_{\varepsilon}\right\|_{1}}{\mu-\left\|G_{\varepsilon}\right\|_{1}}\left\|S_{1}-S_{2}\right\|_{C_{\mu}^{+}\left(Y^{\alpha}\right)}
$$

It means that $(U, S) \in C_{\mu}^{+}$is a solution of (3.14) iff $U=h(S)$ and $S$ solves the equation

$$
S(t)=\exp (-A t / \varepsilon) \xi+\frac{1}{\varepsilon} \int_{0}^{t} \exp (-A(t-s) / \varepsilon) f(S)(s) \mathrm{d} s=\mathfrak{F}^{\mathrm{S}}(\xi, S)
$$

for any $t \geq 0$, where $f(S)(s):=F_{\varepsilon}(\bar{U}(s)+h(S)(s), \bar{S}(s)+S(s))-F_{\varepsilon}(\bar{U}(s), \bar{S}(s))$. Since

$$
\begin{aligned}
\left\|f\left(S_{1}\right)-f\left(S_{2}\right)\right\|_{C_{\mu}^{+}(Y)} & \leq\left\|F_{\varepsilon}\right\|_{1}\left\|h\left(S_{1}\right)-h\left(S_{2}\right)\right\|_{C_{\mu}^{+}(X)}+\left\|D_{S} F_{\varepsilon}\right\|\left\|S_{1}-S_{2}\right\|_{C_{\mu}^{+}\left(Y^{\alpha}\right)} \\
& \leq\left(\left\|F_{\varepsilon}\right\|_{1}\left\|G_{\varepsilon}\right\|_{1}\left(\mu-\left\|G_{\varepsilon}\right\|_{1}\right)^{-1}+\left\|D_{S} F_{\varepsilon}\right\|\right)\left\|S_{1}-S_{2}\right\|_{C_{\mu}^{+}\left(Y^{\alpha}\right)}
\end{aligned}
$$

the mapping $S \mapsto \mathcal{F}^{S}(\xi, S)$ is a uniform contraction on $C_{\mu}^{+}\left(Y^{\alpha}\right)$ with respect to $\xi \in Y^{\alpha}$, provided that $\mu \gg 1$ is large enough and $\left\|D_{S} F_{\varepsilon}\right\| \leq \delta \ll 1$ is sufficiently small for $\varepsilon \in\left(0, \varepsilon_{0}\right], \varepsilon_{0} \ll 1$.

For a given $\xi \in Y^{\alpha}$, we denote $S^{\xi} \in C_{\mu}^{+}\left(Y^{\alpha}\right)$ the unique fixed point of $S=\mathscr{F}^{S}(\xi, S)$. Again, due to the parameterized contraction principle the mapping $\xi \mapsto S^{\xi}$ is Lipschitzian and, hence, the mapping $Y^{\alpha} \ni \xi \mapsto\left(U^{\xi}, S^{\xi}\right) \in C_{\mu}^{+}\left(X \times Y^{\alpha}\right), U^{\xi}:=h\left(S^{\xi}\right)$, is Lipschitz continuous as well. Finally, if we denote

$$
g(\xi):=\bar{U}(0)+U^{\xi}(0), \quad \xi \in Y^{\alpha},
$$


then the mapping $g: Y^{\alpha} \rightarrow X$ is also Lipschitz continuous. We recall that $\left(U^{*}(0), S^{*}(0)\right) \in \mathfrak{M}_{\varepsilon}$ iff $S^{*}(0)=\Phi_{\varepsilon}\left(U^{*}(0)\right)$. However, the last condition is satisfied if and only if

$$
\xi=S^{*}(0)-\bar{S}(0) \in Y^{\alpha}
$$

is a solution of

$$
\Phi_{\varepsilon}(g(\xi))-\bar{S}(0)=\xi .
$$

Now, if we suppose that $A^{-1}: Y \rightarrow Y$ is a compact linear operator than, by [1, chapter 1] the embedding $Y^{\beta} \hookrightarrow \hookrightarrow Y^{\alpha}$ is compact whenever $\alpha<\beta$. We then claim that the mapping $X \ni x \mapsto \Phi_{\varepsilon}(x) \in Y^{\alpha}$ has a compact range. Indeed, by lemmas 3.2 and 3.3 we know that

$$
\Phi_{\varepsilon}(x)=\frac{1}{\varepsilon} \int_{-\infty}^{0} \exp (A s / \varepsilon) F_{\varepsilon}\left(U_{\varepsilon}(x)(s), \phi_{\varepsilon}\left(U_{\varepsilon}(x)\right)(s)\right) \mathrm{d} s .
$$

This yields the estimate

$$
\left\|\Phi_{\varepsilon}(x)\right\|_{Y^{\beta}} \leq K_{0}\left\|F_{\varepsilon}\right\|_{0} \varepsilon^{-1} \int_{-\infty}^{0}(-S / \varepsilon)^{-\beta} \mathrm{e}^{\omega s / \varepsilon} \mathrm{d} s=: K_{0}(\beta)<\infty \quad \text { for any } x \in X
$$

for any $\alpha \leq \beta<1$. Then the mapping $Y^{\alpha} \ni \xi \mapsto \Phi_{\varepsilon}(g(\xi))-\bar{S}(0) \in Y^{\alpha}$ is compact and Lipschitz continuous. Moreover, it takes a ball $B(0, R) \subset Y^{\alpha}$ into itself, $R=K_{0}(\alpha)+\|\overline{S(0)}\|_{Y^{\alpha}}$. Due to the Schauder fixed point theorem there is a solution $\xi \in Y^{\alpha}$ of (3.16). In other words, there exists a $\left(U^{*}(0), S^{*}(0)\right) \in \mathfrak{T}_{\varepsilon}, U^{*}(0)=g(\xi), S^{*}(0)=\bar{S}(0)+\xi$, such that $\left\|\bar{U}(t)-U^{*}(t)\right\|_{X}+$ $\left\|\bar{S}(t)-S^{*}(t)\right\|_{Y^{\alpha}}=O\left(\mathrm{e}^{-\mu t}\right)$ when $t \mapsto \infty$. It completes the proof of lemma 3.5.

Remark 3.6. In case the Lipschitz constants of $\Phi_{\varepsilon}$ and $g$ are less than 1, equation (3.16) can be solved by means of the Banach fixed point theorem (see, [3, theorem 5.1]). Since we have provided no bounds on the Lipschitz constant of $\Phi_{\varepsilon}$ we cannot apply a contraction principle here. This is why we have to turn to Schauder's fixed point principle and therefore the compactness of $A^{-1}$ is needed in our proof.

In the following we will show that this family of fixed points $U_{\varepsilon}(x)$ and their derivatives $D_{x} U_{\varepsilon}(x)$ depend continuously on $\varepsilon>0$ when $\varepsilon$ tends to $0^{+}$uniformly w.r. to $x \in B$, where $B \subset X$ is an arbitrary bounded subset.

The proof uses abstract results due to Mora and Solà-Morales [6] regarding the limiting behavior of fixed points of a two-parameter family of nonlinear mappings. The main difficulty is that the mapping $(U, S) \mapsto\left(G_{\varepsilon}(U, S), F_{\varepsilon}(U, S)\right)$ from the space $C_{\mu, \rho, a}^{-}\left(X \times Y^{\alpha}\right)$ into $C_{\mu, \rho, u}^{-}(X \times Y)$ need not be generally $C^{1}$ differentiable and, therefore, $T_{\varepsilon}(x, \cdot): C_{\mu}^{-}(X) \rightarrow$ $C_{\mu}^{-}(X)$ need not be $C^{1}$ as well. According to lemma 3.1 one can, however, expect that it becomes differentiable after composition with an embedding operator $J_{\mu, \kappa}$ for some $0<\mu<\kappa$. This is why we need a version of a contraction theorem covering the case in which differentiability involves a pair of Banach spaces.

Consider a two parameter family of mappings $T_{\varepsilon}(x, \cdot): \mathcal{U} \rightarrow \mathcal{U}, \varepsilon \in\left[0, \varepsilon_{0}\right], x \in X$, where $X$ is a Banach space. We assume that the Banach space $U$ is continuously embedded into a Banach space $U$ through a linear embedding operator $J$. We also denote $T_{\varepsilon}:=J T_{\varepsilon}$ and $\bar{U}_{\varepsilon}(x):=J U_{\varepsilon}(x)$.

Now a slightly modified version of $[6$, theorem 5.1$]$ reads as follows. 
THEOREM 3.7 [8, theorem 3.6]. Besides the hypothesis (T) we assume also that the mappings $\bar{T}_{\varepsilon}: X \times \mathcal{u} \rightarrow \bar{u}, \varepsilon \in\left[0, \varepsilon_{0}\right]$ satisfy the following conditions:

(1) for any $\varepsilon \in\left[0, \varepsilon_{0}\right], \bar{T}_{\varepsilon}$ is Fréchet differentiable with $D \bar{T}_{\varepsilon}: X \times \mathcal{U} \rightarrow L(X \times \mathcal{U}, \overline{\mathcal{U}})$ bounded and uniformly continuous and there exist mappings

$$
d_{U} T_{\varepsilon} ; X \times \mathcal{u} \rightarrow L(\mathcal{u}, \mathcal{u}) ; \quad \bar{d}_{U} T_{\varepsilon}: X \times \mathcal{u} \rightarrow L(\overline{\mathcal{u}}, \overline{\mathcal{u}}) ; \quad d_{x} T_{\varepsilon}: X \times \mathcal{u} \rightarrow L(X, \mathcal{u})
$$

such that

$$
\begin{gathered}
D_{U} \bar{T}_{\varepsilon}(x, U)=J d_{U} T_{\varepsilon}(x, U)=\bar{d}_{U} T_{\varepsilon}(x, U) J, \quad D_{x} \bar{T}_{\varepsilon}(x, U)=J d_{x} T_{\varepsilon}(x, U) \\
\left\|d_{U} T_{\varepsilon}(x, U)\right\|_{L(\mathrm{u}, \mathrm{u})} \leq \theta, \quad\left\|\bar{d}_{U} T_{\varepsilon}(x, U)\right\|_{L(\overline{\mathrm{u}}, \overline{\mathrm{u}})} \leq \theta, \quad\left\|d_{x} T_{\varepsilon}(x, U)\right\|_{L(X, \mathrm{u})} \leq Q ;
\end{gathered}
$$

(2) for any $B$ bounded and open subset of $X, D \bar{T}_{\varepsilon}(x, U) \rightarrow D \bar{T}_{0}(x, U)$ as $\varepsilon \rightarrow 0^{+}$uniformly for $(x, U) \in\left\{\left(x, U_{\varepsilon}(x)\right), x \in B, \varepsilon \in\left[0, \varepsilon_{0}\right]\right\}$.

Then the mappings $\bar{U}_{\varepsilon}: X \rightarrow \overline{\mathcal{u}}$ have the following properties:

(a) for any $\varepsilon \in\left[0, \varepsilon_{0}\right] ; \bar{U}_{\varepsilon}: X \rightarrow \overline{\mathcal{U}}$ is Fréchet differentiable, with $D \bar{U}_{\varepsilon}: X \rightarrow L(X, \overline{\mathcal{U}})$ bounded and uniformly continuous,

(b) for any $B$ bounded and open subset of $X, D \bar{U}_{\varepsilon}(x) \rightarrow D \bar{U}_{0}(x)$ as $\varepsilon \rightarrow 0^{+}$uniformly with respect to $x \in B$.

In order to apply theorem 3.7 we choose the Banach spaces defined in (3.13). The space $\mathcal{U}$ is continuously embedded into $\overline{\mathcal{U}}$ through the linear embedding operator

$$
J=J_{\mu, \kappa}: C_{\mu, \rho, a}^{-}(X)=\mathcal{u} \rightarrow C_{\kappa, \rho, a}^{-}(X)=\overline{\mathcal{u}} .
$$

If we suppose that the assumptions of lemma 3.2 are satisfied then the mapping $\phi_{\varepsilon}$ is well defined and, hence, we can introduce the mapping $\mathcal{G}_{\varepsilon}: u \rightarrow C_{\mu}^{+}(X)$

$$
\mathcal{S}_{\varepsilon}(U)(s):=G_{\varepsilon}\left(U(s), \phi_{\varepsilon}(U)(s)\right) \quad \text { for any } U \in \mathcal{U} \text { and } s \leq 0 .
$$

Now assume that $B \subset X$ is a bounded subset and define the set

$$
\mathcal{B}_{B}:=\left\{U_{\varepsilon}(x), x \in B, \varepsilon \in\left[0, \varepsilon_{0}\right]\right\} .
$$

Since $U_{\varepsilon}(x)=T_{\varepsilon}\left(x, U_{\varepsilon}(x)\right)=x+J \mathcal{G}_{\varepsilon}\left(U_{\varepsilon}(x)\right)$ and both $\mathcal{G}_{\varepsilon}$ and $\mathfrak{J}$ are bounded, we obtain

$$
B_{B} \text { is a bounded subset of } \mathcal{U} \text {. }
$$

Lemmas 2.1 and 3.2 enables us to conclude that

$$
\bar{S}_{\varepsilon}:=J_{\mu, \kappa} S_{\varepsilon} \in C_{b d d}^{1}\left(\mathcal{U}, C_{\kappa}^{-}(X)\right), \quad \varepsilon \in\left[0, \varepsilon_{0}\right]
$$

and there exists a mapping $d \mathcal{S}_{\varepsilon}: \mathfrak{u} \rightarrow L\left(\mathcal{u}, C_{\mu}^{-}(X)\right)$ such that $D \overline{\mathcal{S}}_{\varepsilon}=J_{\mu, \kappa} d \mathcal{G}_{\varepsilon}$,

$$
d \mathcal{S}_{\varepsilon}(U) W=D_{U} G_{\varepsilon}\left(U(\cdot), \phi_{\varepsilon}(U)(\cdot)\right) W+D_{S} G_{\varepsilon}\left(U(\cdot), \phi_{\varepsilon}(U)(\cdot)\right) d \phi_{\varepsilon}(U) W
$$

We also remind ourselves that $\mathcal{U} \hookrightarrow C_{\mu}^{-}(X)$ and so $\phi_{\varepsilon} \in C_{b d d}^{1}\left(\mathcal{U}, C_{(1+\eta) \mu}^{-}\left(Y^{\alpha}\right)\right)$, $D \phi_{\varepsilon}=$ $J_{\mu,(1+\eta) \mu} d \phi_{\varepsilon}$ (see lemma 3.2(iii)). 
Lemma 3.8. $D \overline{\mathcal{G}}_{\varepsilon}(U) \rightarrow D \overline{\mathcal{G}}_{0}(U)$ as $\varepsilon \rightarrow 0^{+}$uniformly with respect to $U \in \mathbb{B}_{B}$.

Proof. First observe that

$$
D \overline{\mathcal{Q}}_{\varepsilon}(U) W=J_{\mu, \kappa} D_{U} G_{\varepsilon}\left(U(\cdot), \phi_{\varepsilon}(U)(\cdot)\right) W+J_{(1+\eta) \mu, \kappa} D_{S} G_{\varepsilon}\left(U(\cdot), \phi_{\varepsilon}(U)(\cdot)\right) D \phi_{\varepsilon}(U) W
$$

for any $U \in \mathcal{O}_{B}$ and $W \in \mathcal{U}$. By lemma 3.2(ii), we know that $\lim _{\varepsilon \rightarrow 0^{+}}\left(U, \phi_{\varepsilon}(U)\right)=\left(U, \phi_{0}(U)\right)$ in $C_{\mu}^{-}\left(X \times Y^{\alpha}\right)$ uniformly w.r. to $U \in \mathcal{B}_{B}$. According to lemma $2.1, \tilde{G}_{\varepsilon} \in C_{b d d}^{1}\left(C_{\nu}\left(X \times Y^{\alpha}\right)\right.$, $\left.C_{\kappa}(X)\right)$ for $v=\mu$ or $v=(1+\eta) \mu$ and $D \tilde{G}_{\varepsilon}\left(U, \phi_{\varepsilon}(U)\right) \rightarrow D \tilde{G}_{0}\left(U, \phi_{0}(U)\right)$ as $\varepsilon \rightarrow 0^{+}$ uniformly w.r. to $U \in \beta_{B}$. Now the proof of lemma follows from the fact that $D \tilde{G}_{\varepsilon}(U, S)=$ $J_{v, \kappa} D G_{\varepsilon}(U, S)$.

Now we are in a position to apply theorem 3.7 to the family of operators $\left\{T_{\varepsilon}\right\}$. To do so we define the following operators

$$
d_{U} T_{\varepsilon}: X \times \mathcal{u} \rightarrow L(\mathcal{u}, \mathfrak{u}), \quad d_{x} T_{\varepsilon}: X \times \mathcal{u} \rightarrow L(X, \mathcal{u}), \quad \bar{d}_{U} T_{\varepsilon}: X \times \mathcal{u} \rightarrow L(\overline{\mathcal{u}}, \overline{\mathcal{u}})
$$

as follows

$$
d_{U} T_{\varepsilon}(x, U):=J d \mathrm{~S}_{\varepsilon}(U) ; \quad d_{x} T_{\varepsilon}(x, u):=I_{X} ; \quad \bar{d}_{U} T_{\varepsilon}(x, U):=\bar{\jmath} \bar{d} \mathrm{~S}_{\delta}(U),
$$

where the linear operators $\mathfrak{J} \in L\left(C_{\mu}(X), \mathcal{U}\right)$ and $\bar{\jmath} \in L\left(C_{\kappa}(X), \overline{\mathcal{U}}\right)$ were introduced in (3.12), $v=\mu$ or $v=\kappa$, respectively. Furthermore, if we denote

$$
\bar{T}_{\varepsilon}:=J_{\mu, \kappa} T_{\varepsilon}: X \times \mathcal{u} \rightarrow \bar{u} \quad \text { and } \quad \bar{U}_{\varepsilon}(x):=J_{\mu, \kappa} U_{\varepsilon}(x)
$$

then we obtain from (3.18), (3.19) and lemma 3.5,

$$
\bar{T}_{\varepsilon} \in C_{b d d}^{1}(X \times \mathcal{U}, \overline{\mathcal{u}}) \quad \text { and } \quad \lim _{\varepsilon \rightarrow 0^{+}} D \bar{T}_{\varepsilon}(x, U)=D \bar{T}_{0}(x, U)
$$

uniformly for $(x, U) \in\left\{\left(x, U_{\varepsilon}(x)\right), x \in B, \varepsilon \in\left[0, \varepsilon_{0}\right]\right\}$. Under the assumptions of lemma 3.5 we also know that the mappings $d_{U} T_{\varepsilon}, d_{x} T_{\varepsilon}$ and $\bar{d}_{U} T_{\varepsilon}$ satisfy all the hypotheses of theorem 3.7 with some $0<\theta<1$ and $Q=1$, provided that $\mu \gg 1$ is large enough.

Finally, we recall that the mapping $\Phi_{\varepsilon}$ was defined as $\Phi_{\varepsilon}(x)=\phi_{\varepsilon}\left(U_{\varepsilon}(x)\right)(0)$ (see lemma 3.5). With regard to theorem 3.7 and lemma 3.2(iii), (iv); the mapping $X \ni x \mapsto \phi_{\varepsilon}\left(U_{\varepsilon}(x)\right) \in C_{\bar{\mu}}^{-}\left(Y^{\alpha}\right)$ becomes $C_{b d d}^{1}$ differentiable, for some $\bar{\mu}>\kappa$, and $\phi_{\varepsilon}\left(U_{\varepsilon}(x)\right) \rightarrow \phi_{0}\left(U_{0}(x)\right), x \in B$, as $\varepsilon \rightarrow 0^{+}$in the respective topology. Hence, $\Phi_{\varepsilon} \rightarrow \Phi_{0}$ as $\varepsilon \rightarrow 0^{+}$in $C_{h d d}^{1}\left(B, Y^{\alpha}\right)$ where $B \subset X$ is arbitrary bounded open subset.

Summarizing all the preceding results we can state the main result of this paper.

ThForem 3.9. Assume that the hypothesis $(\mathrm{H})$ is fulfilled. Then there are constants $\delta>0$ and $0<\varepsilon_{1} \leq \varepsilon_{0}$ such that if $\left\|D_{S} F_{\varepsilon}\right\|_{L\left(Y^{\alpha}, Y\right)} \leq \delta$ for any $\varepsilon \in\left[0, \varepsilon_{1}\right]$ then there exists an invariant manifold $\mathfrak{M}_{\varepsilon}$ for the semi-flow $S_{\varepsilon}$ generated by the system of evolutionary equations (1.1),

$$
\begin{gathered}
\mathfrak{M}_{\varepsilon}=\left\{\left(U, \Phi_{\varepsilon}(U)\right), U \in X\right\}, \quad \text { where } \Phi_{\varepsilon} \in C_{b d d}^{1}\left(X, Y^{\alpha}\right), \\
\Phi_{\varepsilon} \rightarrow \Phi_{0} \quad \text { as } \varepsilon \rightarrow 0^{+} \text {in } C_{b d d}^{1}\left(B, Y^{\alpha}\right)
\end{gathered}
$$

for any bounded open subset $B \subset X$. 
If $\operatorname{dim}(X)=\infty$ then $\mathfrak{K}_{\varepsilon}$ is infinite dimensional Banach submanifold of the phase-space $X \times Y^{\alpha}$. If $\operatorname{dim}(Y)=\infty$ then $\operatorname{codim}\left(\mathfrak{M}_{\varepsilon}\right)=\infty$.

If, in addition, the resolvent operator $A^{-1}: Y \rightarrow Y$ is compact then the manifold $\mathfrak{N}_{\varepsilon}$, $\varepsilon \in\left(0, \varepsilon_{1}\right]$ is also exponentially attractive, i.e. $\mathfrak{N}_{\varepsilon}$ attracts exponentially any bounded subset of $X \times Y^{\alpha}$.

Remark 3.10. One may ask whether the assumption that the $F_{\varepsilon}$ and $G_{\varepsilon}$ are globally bounded in the respective topologies is not too much restrictive from the point of view of possible applications of the results obtained in theorem 3.9. In case of dissipative semi-flows one can, however, prepare the nonlinearities $F_{\varepsilon}, G_{\varepsilon}$ in such a way that they are vanishing far from the vicinity of a globally attracting set (see, e.g. $[4,6,8]$ ). In Section 4 we present an example of such a modification of the governing equations. Let us also emphasize that having modified the nonlinearities in (1.1) by their truncation we are afterwards dealing with local invariant manifolds only.

\section{AN APPLICATION TO THE JOHNSON-SEGALMAN-OLDROYD MODEL OF SHEARING MOTIONS OF A PRESSURE DRIVEN NON-NEWTONIAN FLUID}

Many striking phenomena like spurt or hysteresis were apparently observed in rheological experiments involving the channel flow of highly elastic and very viscous non-Newtonian fluid like some synthesized polymers. The interested reader is referred to the paper by Vinogradov et al. [10] for details. Much effort has been spent to explain such and related phenomena mathematically. In $[9,12,13]$ Nohel et al. have considered the Johnson-Segalman-Oldroyd model of shearing motions of the planar Poisseule flow within a thin channel. The channel is aligned along the $y$ axis and extends between $x \in[-1,1]$. The flow is assumed to be symmetric with respect to $x=0$ and the fluid undergoes the simple shearing. Therefore, we can restrict ourselves to the interval $x \in[0,1]$. Moreover, the flow variables (velocity and stresses) are independent of $y$ so $\mathbf{v}=(0, v(t, x))$. In order to determine extra stress tensor as a functional of the rate of deformation tensor we consider the Johnson-Segalman-Oldroyd constitutive law (see [9]). In nondimensional units the system of partial differential equations governing the motion of such a fluid is a system of parabolic-hyperbolic equations

$$
\begin{aligned}
\sigma_{t} & =-\sigma+(1+z) v_{x} \\
z_{t} & =-z-\sigma v_{x} \\
\varepsilon v_{t} & =v_{x x}+\sigma_{x}+f
\end{aligned}
$$

subject to boundary and initial conditions

$$
\begin{gathered}
v_{x}(t, 0)=v(t, 1)=\sigma(t, 0)=0 \quad \text { for any } t \geq 0 \\
v(0, x)=v_{0}(x), \quad \sigma(0, x)=\sigma_{0}(x), \quad z(0, x)=z_{0}(x) \quad \text { for } x \in[0,1] .
\end{gathered}
$$

We omit here the complete derivation of the initial-boundary value problem (4.1)-(4.2) by referring to [9]. We only remind ourselves that $\sigma$ is the extra stress, $z$ is the difference of normal stresses, $f \in R$ is a constant pressure gradient driving the flow. The parameter $\varepsilon>0$ is proportional to the ratio of the Reynolds number to the Deborah number and is very small compared to other constants in (4.1), $\varepsilon=O\left(10^{-12}\right.$ ) (see [9]). It gives rise to treating $0<\varepsilon \ll 1$ as a small parameter and investigating the singular limiting behavior of inertial manifolds of system (4.1)-(4.2) when $\varepsilon \rightarrow 0^{+}$. 
For the purpose of the analysis, let us introduce the total stress function $S=v_{x}+\sigma+f x$, $x \in[0,1]$. Since the flow is assumed to be symmetric about the centerline the extra shear stress function must be an odd function, i.e. $\sigma(t, 0)=0$. System (4.1)-(4.2) can, therefore, be rewritten as

$$
\begin{aligned}
\sigma_{t} & =-\sigma+(1+z)(S-\sigma-f x) \\
z_{t} & =-z-\sigma(S-\sigma-f x) \\
\varepsilon S_{t}-S_{x x} & =\varepsilon(-\sigma+(1+z)(S-\sigma-f x))
\end{aligned}
$$

subject to boundary and initial conditions

$$
\begin{array}{r}
S(t, 0)=S_{x}(t, 1)=0 \quad \text { for any } t \geq 0 \\
S(0, x)=S_{0}(x), \quad \sigma(0, x)=\sigma_{0}(x), \quad z(0, x)=z_{0}(x) \quad \text { for } x \in[0,1] .
\end{array}
$$

Denote $A S=-S_{x x}$ the selfadjoint operator in $Y=L_{2}(0,1)$ its domain being the Sobolev space $D(A)=\left\{S \in W^{2,2}(0,1), S(0)=S^{\prime}(1)=0\right\}$. The operator $A$ is sectorial in $Y, \operatorname{Re} \sigma(A)>1$ and $A^{-1}: Y \rightarrow Y$ is compact. Moreover, $Y^{1 / 2}=W_{B}^{1,2}=\left\{S \in W^{1,2}(0,1), S(0)=0\right\}$. Let us consider the Banach space $X-\left(L_{\infty}(0,1)\right)^{2}$. The problem (4.3)-(4.4) can be viewed as an abstract problem (1.1) where the nonlinear functions $G(U, S), F_{\varepsilon}(U, S), U=(\sigma, z)$ are defined by the right-hand sidc of (4.3), i.e. $G(U, S)=[-\sigma+(1+z)(S-\sigma-f x),-z-\sigma(S-\sigma-f x)]^{T}$ and $F_{\varepsilon}(U, S)=\varepsilon\{-\sigma+(1+z)(S-\sigma-f x)\}$. Nohel $e t$ al. [12] proved global existence and uniqueness of solutions of the initial-boundary problem (4.3)-(4.4) in the phase-space $X \times Y^{1 / 2}$ (cf. [12]). The inertialess approximation of system (4.3) $)_{\varepsilon}$ when $\varepsilon=0$ yields $S \equiv 0$ and, hence, $(4.3)_{0}$ becomes a system of ordinary differential equations in the Banach space $X=\left(L_{\infty}(0,1)\right)^{2}$

$$
\begin{aligned}
& \sigma_{t}=-\sigma-(1+z)(\sigma+f x) \\
& z_{t}=-z+\sigma(\sigma+f x)
\end{aligned}
$$

extensively studied by Nohel et al. [9, 11, 13].

Let us emphasize that nonlinear functions $F_{\varepsilon}, G$ do not satisfy the assumptions of the hypothesis $(\mathrm{H})$. In fact they are not smoothly bounded functions. Nevertheless, as is usual in similar circumstances (see, e.g. [6]) we will smoothly modify the functions $F_{\varepsilon}, G$ far from the vicinity of some globally attracting bounded set. In what follows, we will seek a bounded attracting set in $X \times Y^{1 / 2}$ independent of $\varepsilon \in\left[0, \varepsilon_{0}\right]$. To do so, let us first multiply the first equation in (4.3) by $\sigma$ and the second one by $1+z$. Their summation then leads to the estimate

$$
\sup _{x \in[0,1]}\left(\sigma^{2}(t, x)+(1+z(t, x))^{2}\right) \leq 1+\mathrm{e}^{-t} \sup _{x \in[0,1]}\left(\sigma_{0}^{2}(x)+\left(1+z_{0}(x)\right)^{2}\right) .
$$

We will let $K_{0}=K_{0}\left(\left\|\sigma_{0}\right\|_{\infty}+\left\|z_{0}\right\|_{\infty}+\left\|S_{0 x}\right\|_{2}\right)$ denote any positive constant increasingly depending on its argument. By $C>0$ we will denote any generic constant independent of $\varepsilon \in\left[0, \varepsilon_{0}\right]$ and initial conditions. From the above inequality it should be obvious that a ball in $X$ of the radius 2 is an attracting set, i.e. for any $\left(\sigma_{0}, z_{0}, S_{0}\right) \in X \times Y^{1 / 2}$ there is $T=T\left(\sigma_{0}, z_{0}\right)>0$ such that $\|\sigma(t, \cdot)\|_{\infty}+\|z(t, \cdot)\|_{\infty} \leq 2$ for every $t \geq T$. Now we observe that

$$
\|-\sigma+(1+z)(S-\sigma-f x)\|_{2} \leq C\left(1+K_{0} \mathrm{e}^{-t}\right)\left(1+\|S\|_{2}\right) \quad \text { for } t \geq 0 .
$$


Taking the inner product in $L_{2}(0,1)$ of the third equation in (4.3) with $-S_{x x}$ we obtain

$$
\begin{aligned}
\frac{\varepsilon}{2} \frac{\mathrm{d}}{\mathrm{d} t}\left\|S_{x}\right\|_{2}^{2}+\left\|S_{x x}\right\|_{2}^{2} & \leq \varepsilon\|-\sigma+(1+z)(S-\sigma-f x)\|_{2}\left\|S_{x x}\right\|_{2} \\
& \leq C \varepsilon\left(1+K_{0} \mathrm{e}^{-t}\right)\left(1+\|S\|_{2}\right)\left\|S_{x x}\right\|_{2} \leq C \varepsilon\left(1+\left\|S_{x x}\right\|_{2}^{2}\right)
\end{aligned}
$$

for any $t \geq K_{0}\left(\sigma_{0}, z_{0}\right)$. Since $\|S\|_{2} \leq\left\|S_{x}\right\|_{2} \leq\left\|S_{x x}\right\|_{2}$ for any $S \in D(A)$ we obtain $\varepsilon \mathrm{d} / \mathrm{d} t\left\|S_{x}\right\|_{2}^{2}+$ $\left\|S_{x}\right\|_{2}^{2} \leq C \varepsilon$ provided that $\varepsilon \in\left[0, \varepsilon_{0}\right]$ and $\varepsilon_{0}$ is small enough. Then

$$
\left\|S_{x}(t, \cdot)\right\|_{2}^{2} \leq\left\|S_{x}(T, \cdot)\right\|_{2}^{2} \exp ((T-t) / \varepsilon)+C \varepsilon \quad \text { for any } t \geq T \text {. }
$$

Furthermore, as the growth of the third equation in $(4.3)_{\varepsilon}$ is only linear in $S$ one can easily prove that the time-one map $\left(\sigma_{0}, z_{0}, S_{0}\right) \mapsto(\sigma(1, \cdot), z(1, \cdot), S(1, \cdot))$ takes bounded sets into bounded sets of the phase-space $X \times Y^{1 / 2}$. This and the above estimates yield bounded dissipativity of the semiflow generated by (4.3)-(4.4). More precisely, there is a constant $R_{0}>0$ independent of $c \in\left[0, \varepsilon_{0}\right]$ and such that, for any bounded set of initial conditions $\leftrightarrow \subset X \times Y^{1 / 2}$ there is a $T=T(\varepsilon, B)>0$ with the property

$$
\|\sigma(t, \cdot)\|_{\infty}^{2}+\|z(t, \cdot)\|_{\infty}^{2}+\left\|S_{x}(t, \cdot)\right\|_{2} \leq R_{0}
$$

for any $\left(\sigma_{0}, z_{0}, S_{0}\right) \in Q$ and $t \geq T$.

Let $\theta \in C_{b d d}^{2}\left(R^{+}, R^{+}\right)$be a smooth cut-off function with the property $\theta \equiv 1$ on $\left[0,2 R_{0}\right]$, $\theta \equiv 0$ on $\left[3 R_{0}, \infty\right)$ and define the modified functions $G^{b}, F_{\varepsilon}^{b}$ as follows

$$
\begin{aligned}
& G^{b}(U, S)(x):=\theta\left(|\sigma(x)|^{2}+|z(x)|^{2}+\|S\|_{\left.W^{1,2}\right) G(U, S)(x)}^{2}\right. \\
& F_{\varepsilon}^{b}(U, S)(x):=\theta\left(|\sigma(x)|^{2}+|z(x)|^{2}+\|S\|_{\left.W^{1,2}\right) F_{\varepsilon}(U, S)(x)}^{2}\right.
\end{aligned}
$$

for a.e. $x \in[0,1]$. Here $U=(\sigma, z) \in X=\left(L_{\infty}(0,1)\right)^{2}$ and $S \in Y^{1 / 2}=W_{B}^{1 / 2}(0,1)$. Note that $W_{B}^{1,2}$ is a Hilbert space its norm squared being two times continuously differentiable and $W_{B}^{1,2} \hookrightarrow L_{\infty}(0,1)$. Recall also that the Nemitzky operator is $C^{2}$ smooth when considered as a function from $L_{\infty}(0,1)$ into itself. Thus

$$
F_{\varepsilon}^{b} \in C_{b d d}^{2}\left(X \times Y^{1 / 2}, Y\right) \quad \text { and } \quad G^{b} \in C_{b d d}^{2}\left(X \times Y^{1 / 2}, X\right) .
$$

For the norm of $D_{S} F_{\varepsilon}^{b}$ we have an estimate $\left\|D_{S} F_{\varepsilon}^{b}\right\|=O(\varepsilon)$ as $\varepsilon \rightarrow 0^{+}$. Since

$$
\mathfrak{K}_{0}=\left\{(U, S), A S=F_{0}(U, S)=0\right\}=\{(U, 0), U \in X\}
$$

we have $\Phi_{0} \equiv 0$. Now we can apply theorem 3.9 to obtain the following theorem.

THEOREM 3.11. There exists an $\varepsilon_{0}>0$ such that, for any $\varepsilon \in\left(0, \varepsilon_{0}\right]$ the nonlinear system of equations (4.3)-(4.4) governing shearing motions of a Poisseule planar flow of the JohnsonSegalman-Oldroyd fluid:

(i) possesses an infinite dimensional local invariant manifold $\mathfrak{M}_{\varepsilon}$ attracting any solution of $(4.3)_{\varepsilon}-(4.4)$;

(ii) there is an $R_{0}>1$ such that any solution of (4.3) $)_{\varepsilon}-(4.4)$ enters a ball of the radius $R_{0}$ in the space $\left(L_{\infty}(0,1)\right)^{2} \times W_{B}^{1 / 2}(0,1)$;

(iii) $\mathfrak{T N}_{\varepsilon}=\left\{\left(\sigma, z, \Phi_{\varepsilon}(\sigma, z)\right),(\sigma, z) \in B_{R_{0}}\right\}, \Phi_{\varepsilon} \in C_{b d d}^{1}\left(B_{R_{0}}, W_{B}^{1,2}(0,1)\right)$, where $B_{R_{0}}=\{(\sigma, z) \in$ $\left(L_{\infty}(0,1)\right)^{2},\|\sigma\|_{\infty}^{2}+\|z\|_{\infty}^{2}<R_{0} \mid$;

(iv) $\Phi_{\varepsilon} \rightarrow 0$ as $\varepsilon \rightarrow 0^{+}$in the topology of the space $C_{b d d}^{1}\left(B_{R_{0}}, W_{B}^{1,2}(0,1)\right)$. 


\section{REFERENCES}

1. HENRY D., Geometric Theory of Semilinear Parabolic Equations, Lecture Notes in Math., Vol. 840. Springer, Berlin (1981).

2. MARION M., Inertial manifolds associated to partly dissipative reaction-diffusion systems, J. math. Analysis Applic. 143, 295 326 (1989).

3. CHOW S.-N. \& LU K., Invariant manifolds for flows in Banach spaces, J. diff. Eqns 74, 285-317 (1988).

4. FOIAS C., SELL G. R. \& TEMAN R., Inertial manifolds for nonlinear evolutionary equations, $J$, diff. Eqns 73, 309-353 (1988).

5. SVIRIDYUK G. A. \& SUKACHEVA T. G., Cauchy problem for a class of semilinear equations of Sobolev type, Sib. mat. J. 31(5), 120-127 (1990). (English translation.)

6. MORA X. \& SOLA-MORALES J., The singular limit dynamics of semilinear damped wave equation, J. diff. Eqns 78, 262-307 (1989).

7. MIKI.AVČIČ M., A sharp condition for existence of an inertial manifold, J. Dyn. diff. Eqns 3, 437-457 (1991).

8. ŠEVC̆OVIČ D., Limiting behaviour of invariant manifolds for a system of singularly perturbed evolution equations, Math. Meth. appl. Sci. 17, 643-666 (1994).

9. MALKUS D. S., NOIIEL J. A. \& PLOIIR B. J., Analysis of new phenomena in shear flow of a non-Newtonian fluid, SIAM J. appl. Math. 51, 899-929 (1991).

10. VINOGRADOV G., MALKIN A., YANOVSKII YU., BORISENKOVA E., YARLYKOV B. \& BEREZHNAYA G., Viscoelastic properties and flow of narrow distribution polybutadienes and polyisoprenes, J. Polymer Sci. A-2 10, 1061-1084 (1972).

11. MALKUS D. S., NOHEL J. A. \& PLOHR B. J., Approximation of piston-driven flows of a non-Newtonian fluid, in Control Theory, Dynamical Systems and Geometry of Dynamics (Edited by ELSWORTHY, EVERITT and LEE), pp. 173-192. Marcel Dekker, New York (1993).

12. NOHEL J. A., PEGO R. L. \& TZAVARAS A., Stability of discontinuous steady states in shearing motion of a non-Newtonian fluid, Proc. R. Soc. Edinb. 115A, 39-59 (1990).

13. NOHEL J. A. \& PEGO R. L., Nonlinear stability and asymptotic behavior of shearing motions of a non-Newtonian fluid, SIAM J. math. Analysis 24, 911-942 (1993).

14. VANDERBAUWHEDE A. \& VAN GILS S. A., Center manifolds and contraction on a scale of Banach spaces, J. funct. Analysis 72, 209-224 (1987). 